\title{
Heterocontrole da fluoretação da água de abastecimento público em cidades do estado de Goiás, Brasil
}

\author{
External control of fluoridation in public water supply systems \\ in the state of Goiás, Brazil
}

\author{
Paulo Sérgio Scalize ${ }^{1}$ \\ Roberta Vieira Nunes Pinheiro ${ }^{1}$ \\ Humberto Carlos Ruggeri Junior ${ }^{2}$ \\ António Albuquerque ${ }^{3}$ \\ Germán Sanz Lobón ${ }^{1}$ \\ Poliana Nascimento Arruda ${ }^{1}$
}

${ }^{1}$ Programas de PósGraduação em Engenharia Ambiental e Sanitária e Ciências Ambientais, Universidade Federal de Goiás. Av. Universitária 1488, Setor Universitário. 74605- 220 Goiânia GO Brasil.

pscalize.ufg@gmail.com

${ }^{2}$ Escola de Engenharia Civil

e Ambiental, Universidade

Federal de Goiás.

${ }^{3}$ Departamento de Engenharia Civil e Arquitetura, Universidade da Beira Interior. Covilhã Portugal.

\begin{abstract}
The addition of fluoride to the public water supply is a method used for reducing tooth decay. In this sense, the control of fluoridation is important for maintaining its efficiency and, at the same time, for avoiding the risk of fluorosis as a result of the consumption of water with excess of fluoride. The objective of this study was to evaluate the adequacy of fluoride levels in the water distributed to populations of the state of Goiás, Brazil. Towards this aim, 5,039 water samples collected between 2011 and 2013 in 225 municipalities of the state of Goiás were analyzed for fluoride level. The results were assessed with regard to season, type of water source and geographic location. Fluoride levels were found to vary between complete absence and $2.5 \mathrm{mg} F / \mathrm{L}$, with $28.2 \%$ of the samples being between 0.6 and $0.8 \mathrm{mg} / \mathrm{L}$, and $39.1 \%$ between 0.55 and $0.84 \mathrm{mg}$ $F / L$. The rainy season produced a greater number of atypical results and higher values than the dry period. The systems supplied by groundwater sources were shown to have a limited control of fluoride concentration compared to systems supplied by surface water sources. Lower concentrations of fluoride were found in samples collected in the North and East Mesoregions of Goias, with less than $7.5 \%$ being between 0.6 and $0.8 \mathrm{mg} \mathrm{F} / \mathrm{L}$, which systems are mainly supplied by groundwater sources with fluoride addition.
\end{abstract}

Key words Fluorosis, Fluoride, Caries, Water treatment
Resumo A adição do flúor na água é um método empregado na redução de cárie dentária. O controle da fluoretação é importante para manter a sua eficiência e, ao mesmo tempo, evitar o risco de ocorrência de fluorose, fruto do consumo de água com flúor em excesso. O objetivo deste trabalho foi avaliar a adequação dos níveis do fluoreto na água distribuída à população do estado de Goiás, Brasil. Foram analisadas 5.039 amostras de água coletadas em 225 municípios de Goiás, entre 2011 e 2013. Os resultados foram analisados por sazonalidade, tipo de manancial e localização geográfica. Ocorreu uma variação entre ausência e 2,5 mg $\mathrm{F} / \mathrm{L}$, com $28,2 \%$ das amostras entre 0,6 e $0,8 \mathrm{mg}$ $\mathrm{F} / \mathrm{L}$ e $39,1 \%$ entre 0,55 e $0,84 \mathrm{mg} \mathrm{F} / \mathrm{L}$. No período de chuva ocorreu um maior número de resultados atípicos e maiores valores quando comparados ao período da seca. Verificou-se que os sistemas abastecidos por água proveniente de mananciais subterrâneos possuem um menor controle de fluoreto quando comparados a sistemas provenientes de mananciais superficiais. Observou-se menores concentrações nas amostras coletadas no Norte $e$ Leste goianos, com menos de 7,5\% entre 0,6 e $0,8 \mathrm{mg} F / L$, para aquelas coletadas de sistemas de abastecimento que utilizam água de manancial subterrâneo com adição de fluoreto, existindo dificuldades na manutenção dos seus teores.

Palavras-chave Fluorose, Fluoreto, Cárie, Tratamento da água 


\section{Introdução}

Durante o último século, a fluoretação tem sido uma medida de saúde pública empregada no controle da cárie dentária em países de todo o mundo, sendo reconhecida como um importante fator para o declínio da prevalência da cárie dentária. As primeiras pesquisas com relação à utilização do fluoreto centraram-se na água, por ser um recurso de fácil acesso à população, entretanto, com o desenvolvimento técnico-científico e a melhoria das condições de higiene buco-dentária, essa prática tem sido reduzida em vários países. Porém, EUA e Brasil mantiveram a prática de adicionar flúor na água destinada ao abastecimento público ${ }^{1}$, que é recomendada devido a características como segurança, facilidade de administração, baixo custo e abrangência populacional, sendo esse um dos meios mais efetivos para manter constante a presença de flúor na cavidade bucal, fundamental para controle da cárie dentária ${ }^{1,2}$. No entanto, entidades como a Organização Mundial da Saúde (OMS) alertam para o risco que concentrações superiores a 1,5 mg F/ $\mathrm{L}^{3,4}$ podem proporcionar, na saúde humana, $\mathrm{o}$ aparecimento de fluorose.

No Brasil, o fluoreto também é adicionado em mais de $90 \%$ dos dentifrícios desde $1989^{5}$, aumentando o risco de fluorose devido a associação da exposição diária ao flúor por essas duas fontes conforme constatado por Lima e Cury $^{6}$. O método da fluoretação da água, apesar dos riscos à saúde, tem-se justificado pelas boas taxas de redução do índice de cárie relatado em mais de 160 trabalhos distribuídos em mais de 10 países ${ }^{7}$. Apesar dos avanços, o Centro-Oeste brasileiro ainda apresenta valores de cárie dentária na dentição permanente atingindo $73 \%$ das crianças de 12 anos e $90 \%$ dos adolescentes de 15 a 19 (Dentes Permanentes Cariados, Perdidos e Obturados - COP-D $\geq 1)^{8}$. Em estudos realizados por Freire et al. ${ }^{9}$, verificou-se ainda um COP-D $\geq 1$ em 64\% das 1947 crianças de 12 anos analisadas no município de Goiânia (GO). Segundo Clarkson e McLoughlin ${ }^{10}$, para maximizar os benefícios e minimizar os riscos, deve existir mecanismos que viabilizem a adição de flúor em uma concentração adequada, exercendo o maior impacto possível na prevenção e controle de cárie, sem aumentar a prevalência de fluorose dental, uma vez que, como observado por Cangussu et al. ${ }^{11}$, as formas mais brandas da fluorose dental ocorrem nos locais onde foram implantados os sistemas de fluoretação e as formas mais severas foram verificadas em mananciais com alto teor de flúor. Deve-se levar em consideração que, no caso brasileiro, alguns municípios não dispõem de meios para adição do flúor ou de mecanismos de vigilância para o controle de sua concentração nos sistemas de abastecimento público. Nesse sentido, o heterocontrole surge como um instrumento importante para o controle do flúor na água, devendo ser aplicado em todas as unidades constituintes do sistema de abastecimento.

Heterocontrole é um termo utilizado em Saúde Pública e, comumente aplicado a estudos relacionados a fluoretação da água ${ }^{12-18}$. Segundo Narvai ${ }^{19}$ o termo heterocontrole foi utilizado pela primeira vez pelo autor em um curso de "Odontologia Preventiva" que foi objeto do VII Congresso Universitário Brasileiro de Odontologia $(\mathrm{CUBO})^{20}$. O heterocontrole é entendido como "o princípio segundo o qual se um bem ou serviço qualquer implica risco ou representa fator de proteção para a saúde pública, então, além do controle do produtor sobre o processo de produção, distribuição e consumo deve haver controle por parte das instituições do Estado". Dessa forma, o objetivo do presente trabalho foi avaliar a adequação dos níveis do fluoreto na água distribuída às cidades do estado de Goiás operadas pela Companhia Estadual de Saneamento (Saneago), considerando o tipo de manancial utilizado para o abastecimento público e a variação sazonal e regional local.

\section{Materiais e métodos}

Segundo dados do Instituto Brasileiro de Geografia e Estatística ${ }^{21}$ o estado de Goiás possui 6.004.045 habitantes em 246 municípios, sendo que destes, 225 municípios têm seus serviços de saneamento operados pela Saneago, os quais foram objeto de estudo e estão apresentados na Figura 1b. O restante dos municípios é de administração pública direta ${ }^{22}$ e não foi considerado nessa pesquisa.

Nos 225 municípios estudados foram coletadas e analisadas 5.039 amostras de água tratada em um período de 24 meses, entre 2011 e 2013. Para estabelecer o número de amostras coletadas em cada município, os mesmos foram segregados em 3 grupos (Figura 1b) considerando suas populações divulgadas em IBGE, conforme também utilizado por Scalize et al. ${ }^{23}$ para avaliação da turbidez na água de abastecimento. O Grupo I é formado por municípios com população superior a 250.000 habitantes, onde as coletas foram realizadas trimestralmente, sendo em cada 

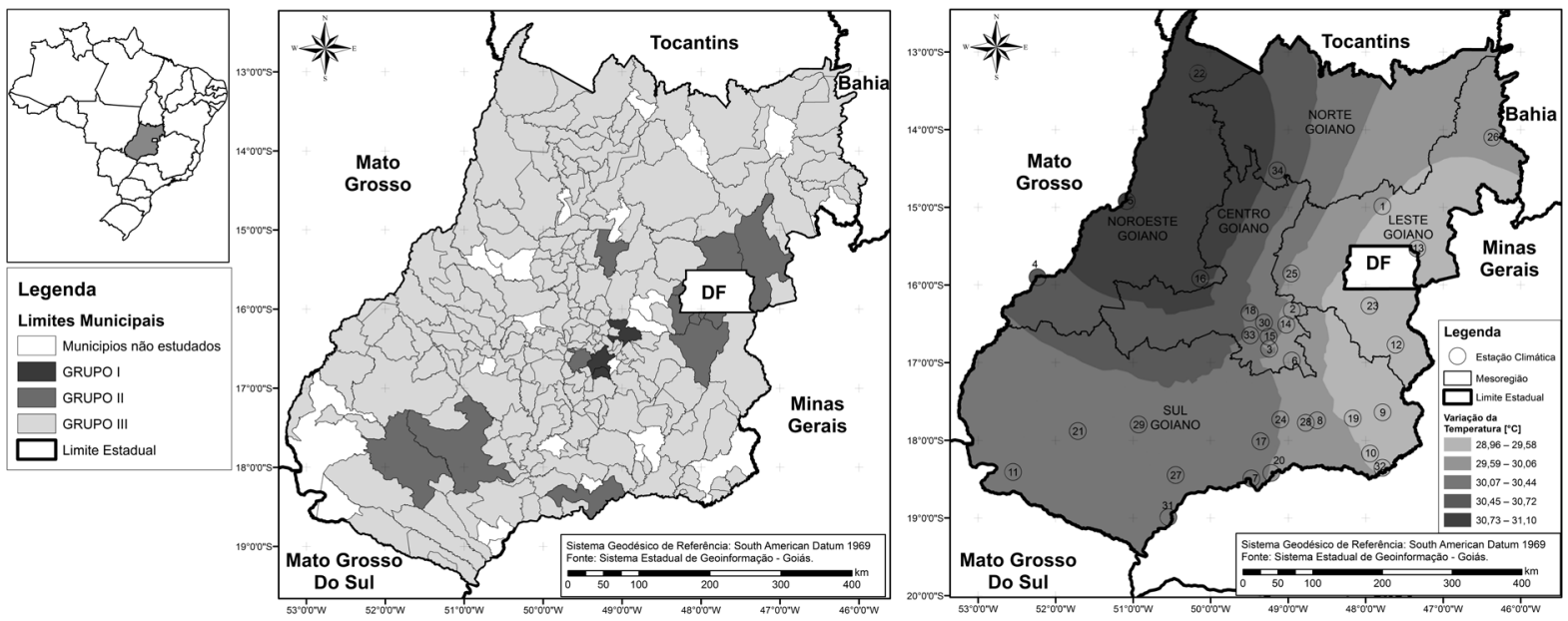

Figura 1. Localização do estado de Goiás no Brasil (a) com a distribuição dos municípios em função da faixa de população divididos em Grupos I, II e III, indicando os municípios do Estado que não foram estudados (b) e a composição das 5 mesorregiões geográficas do Estado contendo as faixas das médias das máximas temperaturas e localização das 34 estações climáticas utilizadas na pesquisa $(\mathrm{c})$.

campanha coletadas de 20 a 30 amostras. Os três municípios integrantes do Grupo I somam 2.092.659 habitantes, o que representa 34,9\% da população do estado de Goiás. O grupo II é formado por municípios com população entre 50.000 e 250.000 habitantes, onde as coletas foram realizadas quadrimestralmente, onde em cada campanha de coleta foram coletadas de 15 a 20 amostras. Os treze municípios integrantes desse grupo somam 1.384.299 habitantes, representado $23,1 \%$ da população total. O terceiro grupo, Grupo III, é formado por municípios com população inferior a 50.000 habitantes, onde as coletas foram realizadas semestralmente, contemplando de 3 a 8 amostras por campanha. Esse grupo apresenta 208 municípios, entretanto a população é inferior a soma das populações pertencentes ao Grupo I e II. Considerando que praticamente não existe um decaimento da concentração do fluoreto na água ao longo da distribuição ${ }^{24}$, a quantidade de amostras foi determinada da mesma forma que Moimaz et al. ${ }^{25}$, considerando a necessidade em coletar ao menos 3 amostras em cada sistema, composta por uma no início da rede de distribuição de água, uma em área intermediária e outra amostra em seu final, de modo a possibilitar uma avaliação do sistema no momento em que a água tratada é disponibilizada para consumo. Outros pontos de coleta foram acrescentados em função do tamanho do município, tendo sido amostrados pontos onde há maior fluxo de pessoas, tais como hospitais, postos de saúde, escolas e creches, conforme estabelecido pela Portaria $n^{\circ} 2.914^{26}$.

As coletas e preservação das amostras foram executadas conforme Guia Nacional de Coleta e Preservação de Amostras ${ }^{27}$, sendo submetidas a uma única análise (análise simples) de Fluoreto empregando o método Spadns, conforme recomendado pelo Standand Methods ${ }^{28}$. Para essa análise foi utilizado um colorímetro da marca Hach, modelo DR 890.

O tratamento dos dados foi realizado considerando o tipo de manancial onde a água foi captada (superficial ou subterrâneo), além de uma análise dos resultados segregados por 5 mesorregiões geográficas, conforme classificação do IBGE regulamentada pela Resolução da Presidência da República (PR) no 11, de 05 de junho de 1990, em que foram incluídas as faixas das médias, das máximas temperaturas e as 34 estações climáticas utilizadas na pesquisa (Figura 1c). Os intervalos das temperaturas foram obtidos a partir dos dados das médias das máximas temperaturas mensais das estações climáticas obtidas do Sistema Estadual de Geoinformação - SIEG ${ }^{29}$. Para o cálculo das isotermas foi determinada a média anual das temperaturas máximas e poste- 
riormente foi aplicado o método de interpolação do Kriging Simple. O resultado obtido serve para ilustrar as diferenças das temperaturas máximas dentro do estado de Goiás, entretanto não pode ser considerado como um modelo geoestatístico certo devido aos poucos pontos disponibilizados e a sua distribuição espacial. O gerenciamento, manejo, tratamento, análise e representação dos dados cartográficos foi realizado utilizando o ArcGis 10.2.

A avaliação dos dados foi realizada considerando-se todos os resultados obtidos utilizandose os valores estabelecidos na Portaria no 635/ GM/MS de 30 de janeiro de $1976^{30}$ que estabelece um teor ótimo de $0,70 \mathrm{mg} \mathrm{F} / \mathrm{L}( \pm 0,10 \mathrm{mg}$ F/L) em função da temperatura média da região, complementando com as faixas propostas por Ramires $^{31}$ e utilizado por Panizzi e Peres ${ }^{32}$ e Ramires et $a{ }^{13}{ }^{13}$, que admitem variação na concentração ótima de fluoreto de $0,15 \mathrm{mg} F / \mathrm{L}$ para o limite inferior e $0,14 \mathrm{mg} F / \mathrm{L}$ para o limite superior, sendo considerado como máximo admissível o teor de 1,50 mg F/L estabelecido pela Portaria MS $2.914^{26}$, que é também o valor aconselhado pela $\mathrm{OMS}^{3}$. Dessa forma, as faixas de teor de fluoreto utilizadas foram: $<0,54 \mathrm{mg} \mathrm{F} / \mathrm{L}=$ Inaceitável; 0,55 mg F/L = Mínimo aceitável; 0,55 a 0,59= Subfluoretada; 0,60 a $0,80=$ Ótimo; 0,81 a $0,84=$ Superfluoretada; 0,84 = Máximo aceitável; 0,85 a 1,5 = Inadequada; $>1,50$ = Inaceitável.

Visando a verificação da interferência sazonal quanto a manutenção do teor de fluoreto na água distribuída a população, os resultados foram analisados considerando dois períodos, chuvoso (janeiro a março e outubro a dezembro) e de estiagem (abril a setembro). Dessa forma, das 5.039 amostras, 2.226 foram coletadas na época da chuva e 2.813 na época da seca.

Os resultados obtidos nas análises de fluoreto foram tratados estatisticamente com XLSTAT, obtendo-se os estatísticos: média, mediana, desvio padrão e o coeficiente de variação, dos quais foram construídos gráficos do tipo boxplot, com um intervalo de confiança de $95 \%$, possibilitando verificar os valores discrepantes de cada parâmetro analisado.

\section{Resultados e discussão}

\section{Análise Geral}

De forma geral, foi encontrada uma grande variação do teor de fluoreto nas amostras analisadas, apresentando uma maior incidência com teores de fluoreto abaixo do mínimo estabelecido para uma efetividade na prevenção da cárie, sendo que apenas $28,2 \%$ das amostras analisadas (Figura 2a) estão com o teor de fluoreto dentro da faixa de 0,60 a $0,80 \mathrm{mg}$ F/L estabelecida pela Portaria no $635 / \mathrm{GM} / \mathrm{MS}^{30}$.

A análise global dos resultados de fluoreto nas 5.039 amostras indicou uma variação em sua concentração, apresentando desde a ausência até concentrações de 2,50 mg F/L, sendo que, valores acima de 1,22 mg F/L foram considerados como pontos atípicos em função da quantidade e do teor de fluoreto encontrado nas amostras avaliadas. A média encontrada para esse parâmetro foi de $0,50 \mathrm{mg}$ F/L com uma mediana de $0,53 \mathrm{mg}$ F/L (Figura 2b). Ficou evidenciado que em 3.617 amostras $(71,8 \%)$, os resultados obtidos para fluoreto foram inferiores ou superiores aos limites da Portaria no $635^{30}$, estando 3.073 amostras (61,0\%) abaixo de $0,60 \mathrm{mg}$ F/L e 544 amostras $(10,8 \%)$ acima de $0,80 \mathrm{mg} \mathrm{F} / \mathrm{L}$. Quando analisados os valores abaixo de $0,55 \mathrm{mg} \mathrm{F} / \mathrm{L}$ e acima de $0,84 \mathrm{mg} \mathrm{F} / \mathrm{L}$, foram encontrados $52,7 \%$ e $8,2 \%$, respectivamente. Foram detectadas ainda $0,60 \%$ de amostras com o teor acima do limite máximo estabelecido pela Portaria $2.914^{26}$, que é de 1,50 mg F/L, alcançando um máximo de 2,5 mg F/L. Segundo Frazão et al. ${ }^{5}$, o limite de 1,50 mg F/L é adotado pela maioria dos países da América do Sul e pela OMS. Peixoto et al. ${ }^{16}$, consideram valores acima de 1,45 como sendo de risco muito alto para o surgimento da fluorose dentária. Esses valores podem ser mais baixos em função do clima da região, uma vez que reflete no consumo de água, como concluído por Craig et al. ${ }^{33}$ em estudo realizado em Gana (temperatura média $29^{\circ} \mathrm{C}$ ), em que foi determinado o limite de $0,60 \mathrm{mg}$ F/L para crianças de 6 a 8 anos, menor que $0,60 \mathrm{mg} F / \mathrm{L}$ para crianças menores de 2 anos e de $1,0 \mathrm{mg}$ F/L para crianças de maior idade e adultos.

O teor médio de fluoreto encontrado foi de $0,50 \mathrm{mg} \mathrm{F} / \mathrm{L}$ (Figura $2 \mathrm{~b}$ ), próximo a média de $0,55 \mathrm{mg} \mathrm{F} / \mathrm{L}$ encontrada por Peixoto et al. ${ }^{16}$, ao avaliarem a concentração de flúor na água de abastecimento do município de Juguaribara-CE, e por Daré et al. ${ }^{34}$ que relatam uma média de 0,57 mg F/L em pesquisa realizada com 5.157 amostras de água na região de Araçatuba-SP. Ramires et al..$^{13}$, analisando 737 amostras de água distribuída a população da cidade de Bauru-SP, encontrou uma concentração média de flúor observada nos diferentes meses de coleta variando entre 0,37 e 1,00 mg F/L, com cerca de $85 \%$ da média das amostras entre 0,55 e $0,84 \mathrm{mg} F / \mathrm{L}$, com um 
(a)

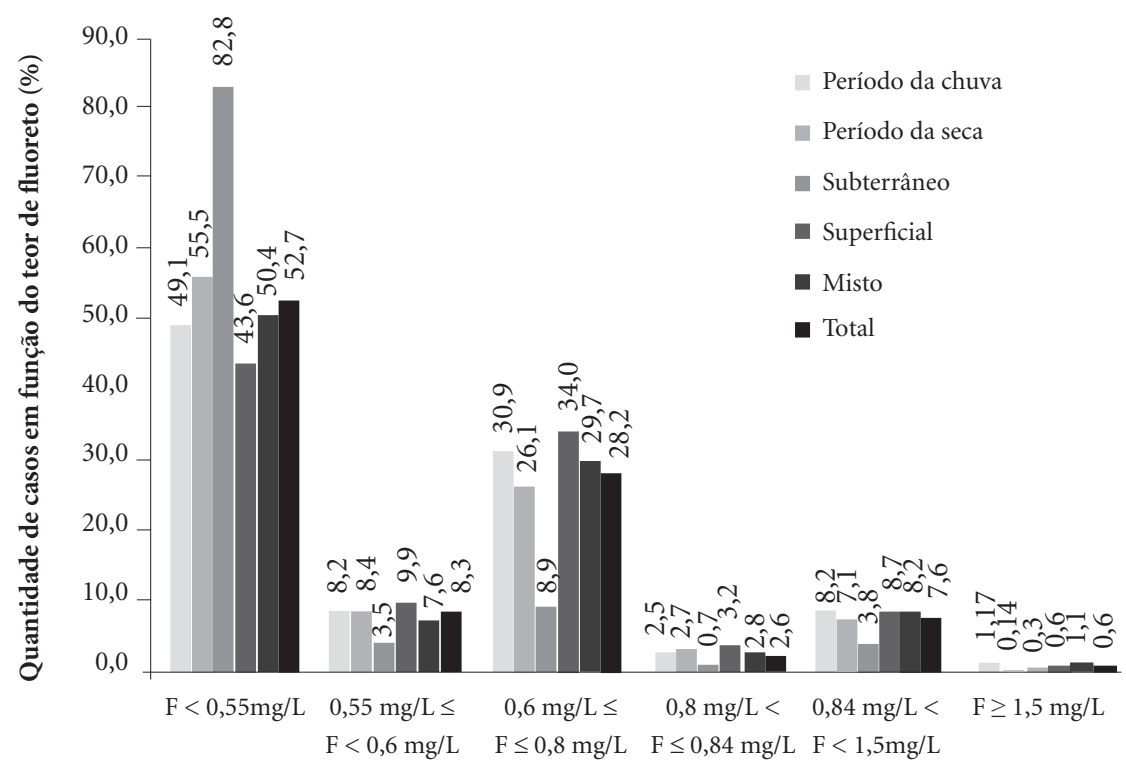

Distribuição em função do teor de fluoreto

(b)

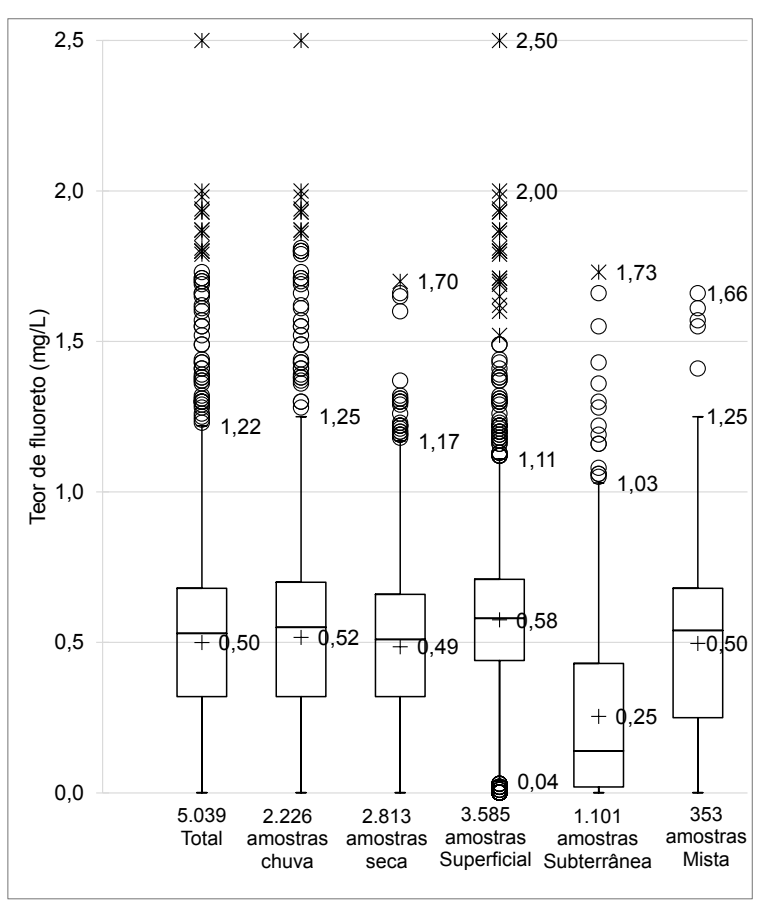

Figura 2. Resultados das análises das amostras, distribuídos em função da concentração de fluoreto e da sazonalidade e do tipo de manancial utilizado para o tratamento.

valor médio de $0,75 \mathrm{mg} \mathrm{F} / \mathrm{L}$. Isso evidencia a dificuldade em se manter a dosagem de fluoreto dentro dos padrões, como também relatado por Leivas et al..$^{35}$ na cidade de Canoas-RS, indicando a necessidade de um maior controle operacional.

\section{Análise quanto ao tipo de manancial e sazonalidade}

Conforme os resultados obtidos nas análises das amostras divididas de forma sazonal (período 
chuvoso e seco), constatou-se que o maior teor de fluoreto predominou nas amostras coletadas no período da chuva, sendo superior nas faixas a partir de 0,60 mg F/L (Figura 2a), resultando uma média de $0,52 \mathrm{mg}$ F/L com pontos atípicos a partir de $1,25 \mathrm{mg} \mathrm{F} / \mathrm{L}$, atingindo um máximo de 2,5 mg F/L (Figura 2b). No período da seca os resultados foram menores, referendando uma média de $0,49 \mathrm{mg} \mathrm{F} / \mathrm{L}$ e pontos atípicos a partir de $1,17 \mathrm{mg} \mathrm{F} / \mathrm{L}$, atingindo um valor máximo de $1,7 \mathrm{mg} \mathrm{F} / \mathrm{L}$ (Figura 2b). No período da chuva, foi encontrado em $1,17 \%$ das amostras um teor de flúor superior ao limite de $1,5 \mathrm{mg} \mathrm{F} / \mathrm{L}$, estabelecido na Portaria $2.914^{26}$. Esse valor é bem superior ao $0,14 \%$ detectado no período da seca (Figura 2a), demonstrando uma maior dificuldade no controle do flúor no período da chuva.

Quanto a distribuição dos resultados da análise realizada em função do tipo de manancial, verificou-se que $86,3 \%$ das amostras analisadas em locais abastecidos por captação subterrânea com adição de fluoreto, apresentaram-se com teor abaixo de 0,60 mg F/L (Figura 2a). Provavelmente isso acontece em decorrência da dificuldade em manter a fluoretação, tendo em vista a necessidade da permanência de um operador de tratamento nesses locais ou algum sistema que permita o seu controle. Já esse problema não ocorre nas Estações de Tratamento de Água (ETAs), que precedem os sistemas de mananciais de águas superficiais visto que possuem constantemente operadores nas estações. No entanto, Lima et al. ${ }^{12}$, verificaram que a constância na concentração mensal do flúor foi quase impossível na maioria das ETAs analisadas.

A falta de treinamento do operador, variações das vazões ao longo da rede de distribuição e problemas nos equipamentos hidráulicos são fatores que contribuem para essas oscilações ${ }^{36}$. Dessa forma, pode ser verificado que $53,5 \%$ das amostras, coletadas em locais provenientes de abastecimento de manancial superficial, estavam com teor de fluoreto abaixo de $0,60 \mathrm{mg}$ F/L e $34 \%$ entre 0,6 e $0,8 \mathrm{mg} \mathrm{F} / \mathrm{L}$, com valor médio de $0,58 \mathrm{mg} \mathrm{F} / \mathrm{L}$ (Figura $2 \mathrm{~b}$ ), que está pouco abaixo dos encontrados por Moimaz et al. ${ }^{25}$, entre 0,61 e 0,66 mg F/L para mananciais superficiais. Já nos sistemas onde existe o abastecimento proveniente de ETA e água subterrânea simultaneamente, há uma mistura dessas águas, seja no interior do reservatório ou na rede de distribuição. Esse procedimento permite um maior controle do teor de fluoreto, o que mantém os teores de fluoretos próximos aos dos sistemas abastecidos somente por água proveniente de ETAs.
Observa-se que, independente da fonte de abastecimento, no período da seca existe uma maior incidência de amostras com teor de fluoreto abaixo de $0,60 \mathrm{mg} \mathrm{F} / \mathrm{L}$, ocorrendo um deslocamento dos valores acima de $0,80 \mathrm{mg} F / \mathrm{L}$ para a faixa entre 0,60 e $0,80 \mathrm{mg} \mathrm{F} / \mathrm{L}$ (Figura 3a). Na Figura $3 b$ pode-se visualizar que no período da seca houve um menor número de resultados atípicos, além de menores valores máximos quando comparados ao período da chuva, sendo que os valores médios aproximaram-se da mediana nas duas situações. Lima et al. ${ }^{12}$, identificaram que as variações na concentração de flúor geralmente ocorrem nas unidades de tratamento e, desta forma, o efeito da temperatura e do tipo de material empregado nas dosagens tende a ser mais significativo do que outros fenômenos físicos, como absorção ou adsorção nas paredes das tubulações do sistema de distribuição.

\section{Análise por mesorregiões considerando tipo de manancial e sazonalidade}

Por sua vez, a análise dos resultados do teor de fluoreto, distribuídos pelas 5 mesorregiões, apontou que as menores concentrações encontradas para as águas tratadas de mananciais superficiais estão nas mesorregiões Norte e Leste goianos com, respectivamente, $60 \%$ e $59,5 \%$ das amostras com concentração abaixo de 0,55 mg F/L (Figura 4a). Essas mesmas mesorregiões também, apresentaram para as águas tratadas de mananciais subterrâneos, uma maior incidência, sendo $86,7 \%$ e $95,1 \%$, respectivamente (Figura 5a). As concentrações médias encontradas para esses mananciais variaram de 0,48 a $0,62 \mathrm{mg} \mathrm{F} / \mathrm{L}$ para águas tratadas de mananciais superficiais (Figura $4 \mathrm{~b}$ ) e entre 0,15 e $0,41 \mathrm{mg} \mathrm{F} / \mathrm{L}$ para águas tratadas de mananciais subterrâneos (Figura $5 b$ ). Cabe destacar que a média mensal das médias das máximas temperaturas diárias registradas na estação climatológica da mesorregião Norte ficaram entre $31,3^{\circ} \mathrm{C}$ (janeiro) e $35,0^{\circ} \mathrm{C}$ (setembro) o que indica um maior volume de água ingerido diariamente, necessitando um menor teor de fluoreto na água. No entanto, o teor ainda se encontra baixo em função do recomendado pela Portaria no 635/GM/MS ${ }^{30}$.

\section{Análise quanto ao tamanho populacional abastecido}

Ao analisar os resultados em função do tamanho da população verificou-se que nos municípios do Grupo I existe uma maior quantidade 
(a)

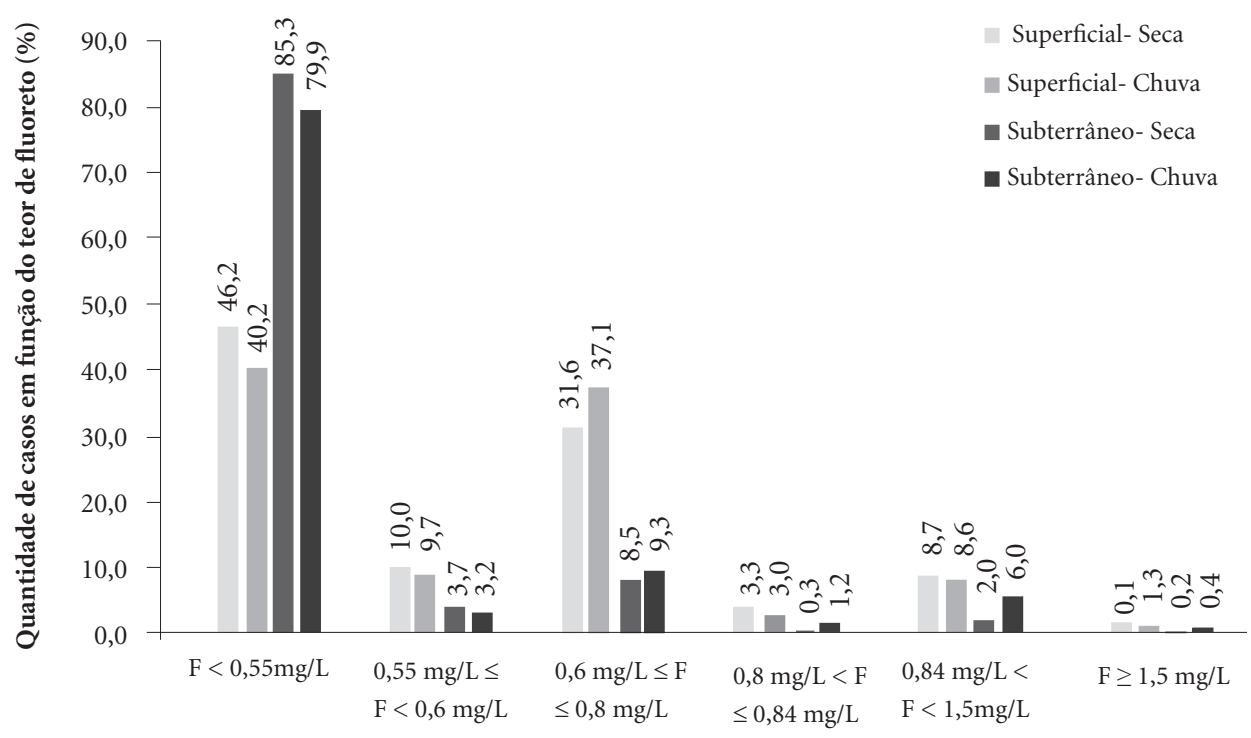

Distribuição em função do teor de fluoreto

(b)

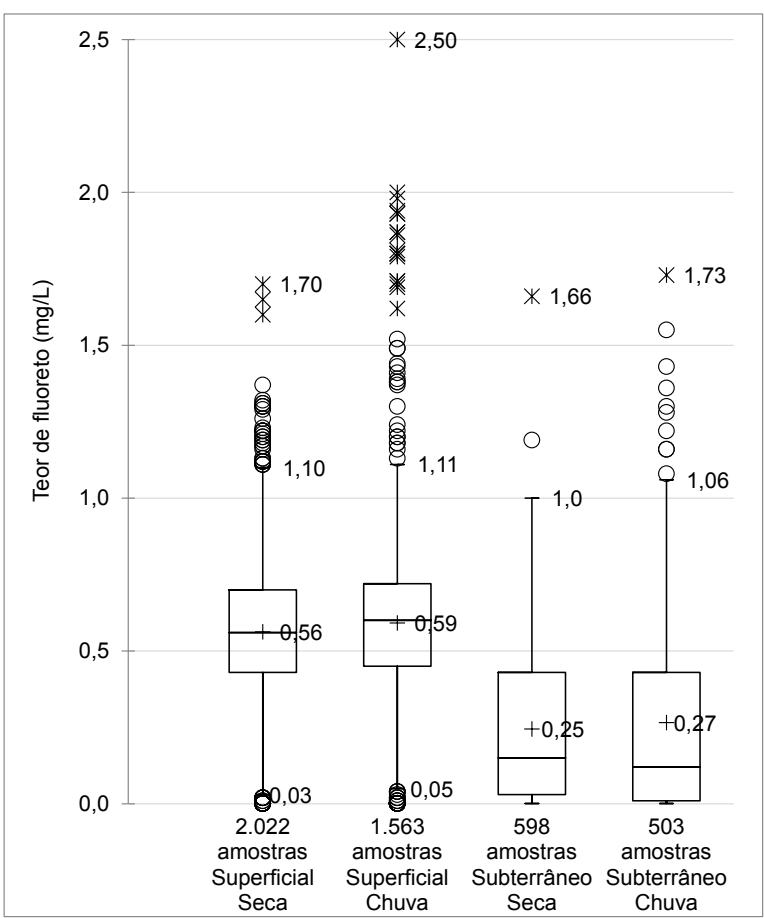

Figura 3. Resultados das análises das amostras, distribuídos em função da concentração de fluoreto, tipo de manancial utilizado para o tratamento e sazonalidade.

de amostras com valores entre 0,60 e $0,80 \mathrm{mg} / \mathrm{L}$ (36,38\%). Nesse Grupo, 49,21\% das amostras estão com os valores entre 0,55 e $0,84 \mathrm{mg} / \mathrm{L}$, enquanto que nos municípios do Grupo II e Grupo
III observou-se $27,43 \%$ e $42,39 \%$, respectivamente. Essa diferença nos valores evidencia uma maior atenção do órgão gestor com os municípios de maior porte. Os municípios do Grupo II 
(a)

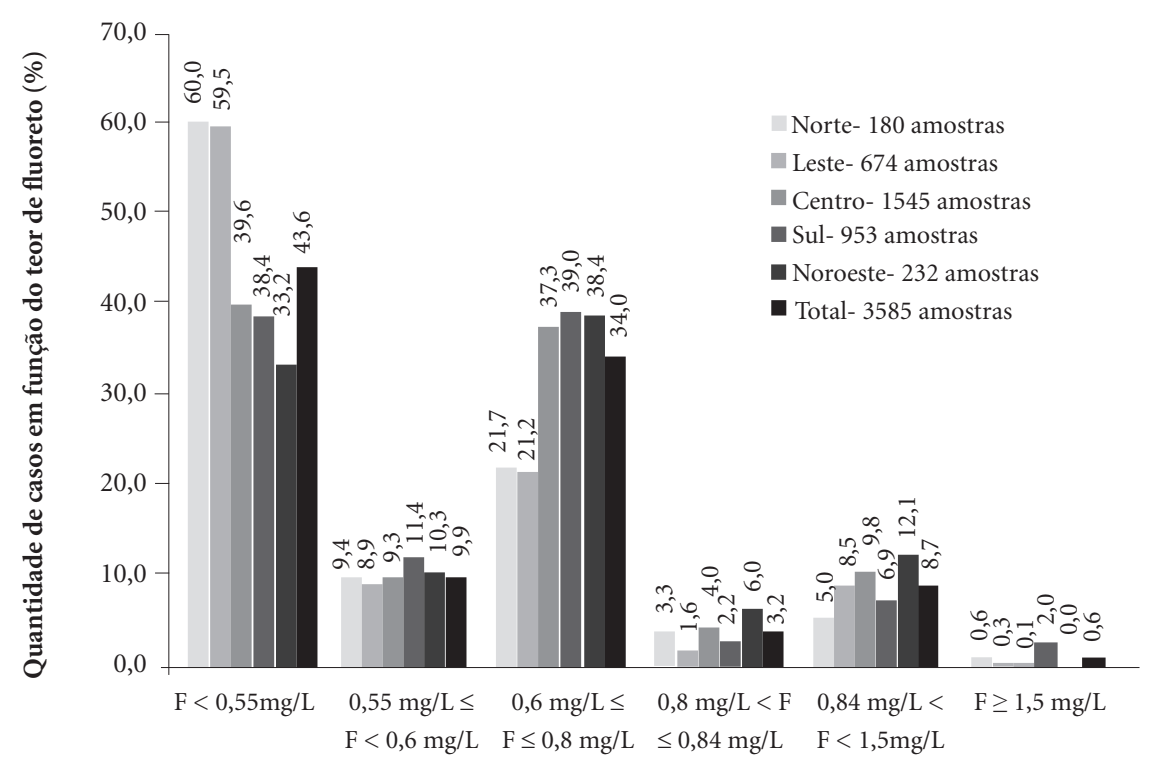

Distribuição em função do teor de fluoreto

(b)

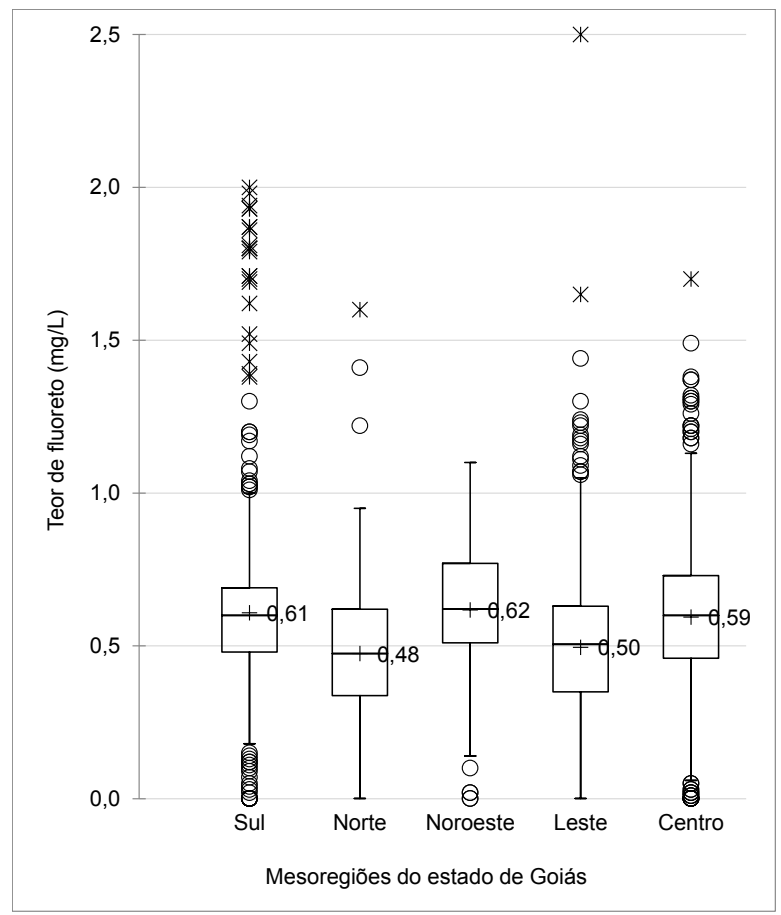

Figura 4. Resultados das análises das amostras coletadas na rede de abastecimento composto por sistema de tratamento de água de mananciais superficiais, distribuídos por mesorregiões e por faixas em função da concentração de fluoreto.

apresentaram uma baixa quantidade de amostras dentro dessa faixa, verificando-se uma quantidade de $61,97 \%$ abaixo de $0,55 \mathrm{mg} / \mathrm{L}$. Esse fato é decorrente de que em alguns municípios desse
Grupo são abastecidos por água de manancial subterrâneo onde não é realizada a fluoretação da água, influenciando nos resultados. 
(a)

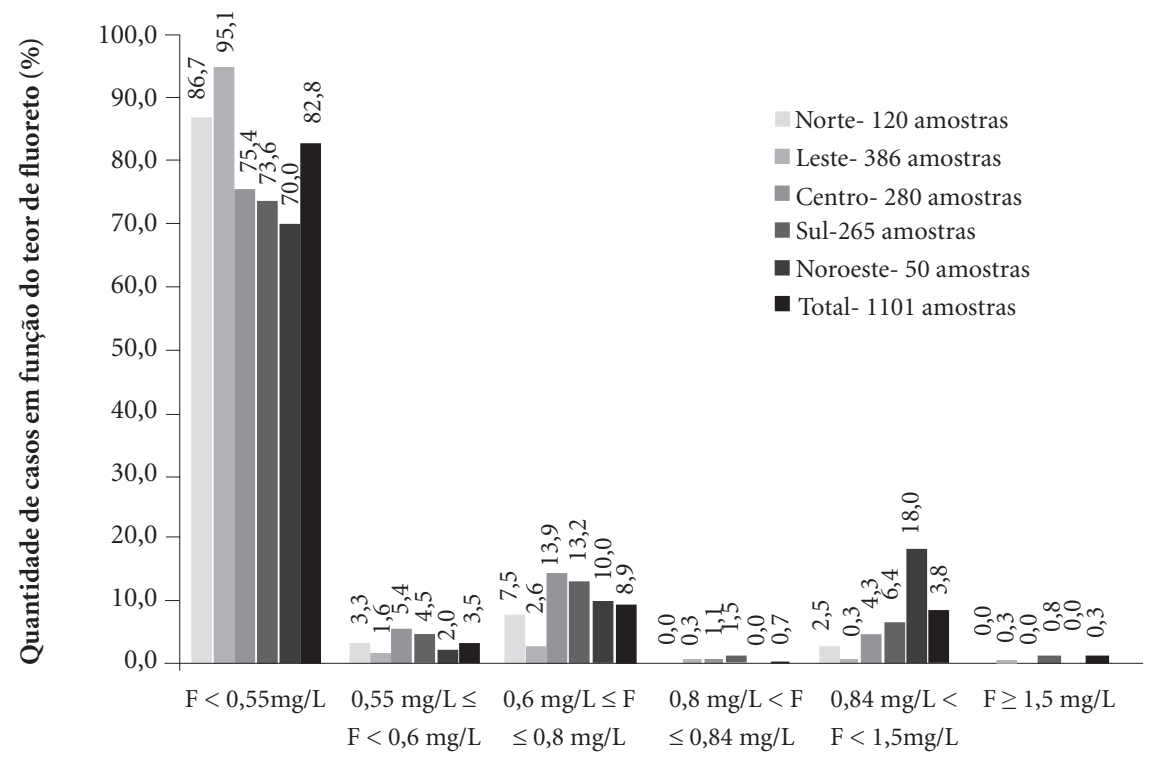

Distribuição em função do teor de fluoreto

(b)

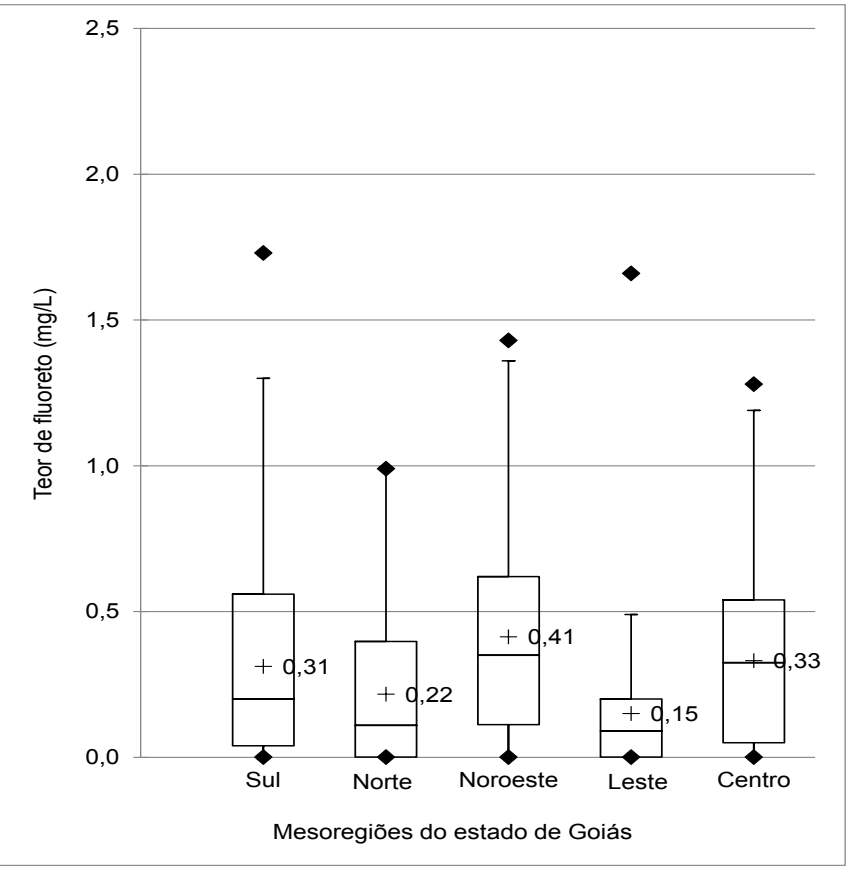

Figura 5. Resultados das análises das amostras coletadas na rede de abastecimento composto por sistema de tratamento de água de mananciais subterrâneos, distribuídos por mesorregiões e por faixas em função da concentração de fluoreto.

\section{Considerações finais}

A maioria dos trabalhos que envolve o acompanhamento das dosagens, descrevem grande variação nas concentrações e este fato dificulta, segundo Ramires e Buzulaf ${ }^{1}$, a avaliação do efeito protetor à cárie e a ocorrência de fluorose na população atendida pelos municípios. Além disso, a distribuição de água com concentração de flúor menor do que a ótima implica desperdício de re- 
curso público e uma prevenção pouco eficiente. Isso mostra que é necessário um heterocontrole efetivo corroborando com Panizzi e Peres ${ }^{32}$, que relata que, após uma intervenção político-jurídica, realizada pelo sistema de vigilância sanitária no município de Chapecó-SC, ocorreram mudanças no sistema operacional e de manutenção refletindo numa melhoria significativa, evoluindo de $26 \%$ para $49 \%$ de amostras com teor de fluoreto dentro da faixa de 0,7 a $0,9 \mathrm{mg} F / L$, a qual é estabelecida pela Portaria no $635 / \mathrm{GM} / \mathrm{MS}^{30}$ para aquela região, que admite variação de $0,10 \mathrm{mg} F / \mathrm{L}$ em função do teor ótimo segundo a faixa de temperatura local. Neste estudo ainda foi relatado o aumento de eficiência de $40 \%$ para $63 \%$ e de $36 \%$ para $61 \%$ caso a faixa fosse de 0,7 a $1,0 \mathrm{mg}$ F/L e de $0,65 \mathrm{mg} \mathrm{F} / \mathrm{L}$ a $0,94 \mathrm{mg} \mathrm{F} / \mathrm{L}$, respectivamente.

O heterocontrole, no estado de Goiás, poderia ser realizado pela Agência Goiana de Regulação (AGR), a qual é responsável pela regulação dos Serviços prestados pela Companhia Estadual de Saneamento (Saneago). Dessa forma, o controle ficaria alinhado com o recomendado por Panizzi e Peres ${ }^{32}$, que sugere a operacionalização por entidades ou instituições públicas, privadas, ou ambas, diferentes das companhias de abastecimento de água.

No entanto, observa-se ainda que a Portaria $\mathrm{n}^{\circ} 635 / \mathrm{GM} / \mathrm{MS}^{30}$ teve como suporte a equação proposta por Galagan e Vermillion ${ }^{37}$, que considerou que as condições de consumo de água pela população brasileira é a mesma da americana, em função somente da média das máximas temperaturas diárias. Nesse contexto, alguns estudos propõem ajustes na equação em função da situação de cada localidade. Ramadan e Hilmi ${ }^{38}$ inseriram um coeficiente de ajuste de 0,56, considerando que a população tem como fonte de líquido apenas a água, descontando a porção (44\%) de líquido proveniente do leite, contabilizado por
Galagan e Vermillion ${ }^{37}$. Isso refletiu num teor de fluoreto ideal para a população do Sudão entre 0,32 e $0,35 \mathrm{mg} F / \mathrm{L}$, diferente do $0,61 \mathrm{mg}$ $\mathrm{F} / \mathrm{L}$ calculado inicialmente apenas considerando a temperatura média máxima local de $35,58^{\circ} \mathrm{C}$. O mesmo foi realizado no Chile por Villa et al..$^{39}$, que onde concluíram que a faixa ideal de 0,50 a 0,60 mg F/L para uma média local das máximas temperaturas variando de 18 a $22^{\circ} \mathrm{C}$.

\section{Conclusão}

O presente trabalho permitiu concluir que:

- existem dificuldades na manutenção das concentrações de fluoreto nos municípios estudados e este fato reforça a necessidade do heterocontrole, sendo que entidades públicas podem auxiliar na regulação para um efetivo controle dos teores de fluoreto em um sistema de abastecimento de água;

- na época sazonal da chuva foi observada uma maior quantidade de amostras com teor de fluoreto superior a $0,60 \mathrm{mg} \mathrm{F} / \mathrm{L}(42,8 \%)$ contra $36 \%$ na época da seca;

- os sistemas abastecidos por água proveniente somente de manancial subterrâneo com adição de fluoreto, foram os que apresentaram menores teores de fluoreto nas águas fornecidas a população;

- existem diferenças no teor de fluoreto encontrado nas amostras de água analisadas levando-se em conta as 5 mesoregiões, sendo encontrados menores valores no Norte e Leste goiano;

- existe a necessidade de estudos mais amplos no intuito de verificar as faixas ideais de fluoreto para o estado de Goiás, visto a íntima ligação com o consumo de água, influenciado pela temperatura, umidade e hábitos higiênico bucal e alimentar. 


\section{Colaboradores}

PS Scalize foi responsável pela concepção do trabalho, confecção dos gráficos, análise e interpretação dos dados. A redação crítica ficou a cargo de A Albuquerque que também contribuiu na discussão dos resultados e conclusão do trabalho. RVN Pinheiro e HC Huggeri Junior foram responsáveis pela revisão da bibliografia e discussão dos dados e conclusão do trabalho. G Sanz Lobon ficou encarregado da elaboração dos mapas temáticos e auxílio no delineamento experimental e também da discussão dos resultados. PN Arruda auxiliou na discussão dos dados.

\section{Referências}

1. Ramires I, Buzulaf MAR. A fluoretação da água de abastecimento público e seus benefícios no controle da cárie dentária: cinquenta anos no Brasil. Cien Saude Colet 2007; 12(4):1057-1065.

2. Cury JA, Tabchoury CPM. Determination of appropriate exposure to fluoride in non-EME countries in the future. J Appl Oral Sci 2003; 11(2):83-95.

3. World Health Organization (WHO). Guidelines for drinking-water quality: recommendations. $3^{\text {th }}$ ed. Geneva: WHO; 2004. v.1.

4. Fawell J, Bailey K, Chilton J, Dahi E, Fewtrell L, Magara Y. Fluoride in drinking water. Geneva: World Health Organization; 2006.

5. Frazão P, Peres MA, Cury JA. Drinking water quality and fluoride concentration. Rev Saude Publica 2011; 45(5):964-973.

6. Lima YBO, Cury JA. Ingestão de flúor por crianças pela água e dentifrício. Rev Saude Publica 2001; 35(6):576581.

7. Rugg-Gunn AJ, Do L. Effectiveness of water fluoridation in caries prevention. Community Dent Oral Epidemiol 2012; 40(s2):55-64.

8. Brasil, Ministério da Saúde (MS). Projeto SB Brasil 2003 - Condições de saúde bucal da população brasileira 2002-2003: resultados principais. Brasília: MS; 2004.

9. Freire MCM, Reis SCGB, Gonçalves MM, Balbo PL, Leles CR. Condição de saúde bucal em escolares de 12 anos de escolas públicas e privadas de Goiânia, Brasil. Rev Panam Salud Publica 2010; 28(2):86-91.

10. Clarkson JJ, McLoughlin J. Role of fluoride in oral health promotion. International Dent J 2000; 50(3):119-128.

11. Cangussu MCT, Narvai PC, Fernandez RC, Djehizian V. A fluorose dentária no Brasil: uma revisão crítica. Cad Saude Publica 2002; 18(1):7-15.

12. Lima FG, Lund RG, Justino LM, Demarco FF, Del Pino FAB, Ferreira R. Vinte e quatro meses de heterocontrole da fluoretação das águas de abastecimento público de Pelotas, Rio Grande do Sul, Brasil. Cad Saude Publica 2004; 20(2):422-429.

13. Ramires I, Maia LP, Rigolizzo DS, Lauris JRP, Buzalaf MAR. External control over the fluoridation of the public water supply in Bauru, SP, Brazil. Rev Saude Publica 2006; 40(5):883-889.

14. Silva JS, Melo do Val C, Costa JN, Moura MS, Silva TAE, Sampaio FC. Heterocontrole da fluoretação das águas em três cidades no Piauí, Brasil. Cad Saude Publica 2010; 23(5):1083-1088.

15. Toassi RFC, Kuhnen M, Cislaghi GA, Bernardo JR. Heterocontrole da fluoretação da água de abastecimento público de Lages, Santa Catarina, Brasil. Cien Saude Colet 2007; 12(3):727-732.

16. Peixoto DF, Alencar KP, Peixoto RF, De Sousa CFM, Sampaio FC, Forte FDS. Heterocontrole da fluoretação da água de abastecimento público do município de Jaguaribara, Ceará, Brasil. Rev Bras Promoç Saude 2012; 25(3):271-277.

17. Vidal SG, Tovo MF, Kramer PF, Ruschel HC, Ferreira EH. Heterocontrole da fluoretação de águas de abastecimento público do município de Torres/RS. Stomatos 2006; 12(22):5-9.

18. Olivati FN, Souza MLR, Tenuta LMA, Cury JA. Quality of drinking water fluoridation of Cienc 2011; 26(4):285-290. 
19. Narvai PC. Fluoretação da água: heterocontrole no Município de São Paulo no período 1990-1999. Rev Bras Odontol Saude Colet 2000; (2):50-6.

20. Narvai PC. Odontologia preventiva. In: Congresso Universitário Brasileiro de Odontologia - CUBO, 7o, São Paulo, 10/09/1982.

21. Instituto Brasileiro de Geografia e Estatística (IBGE). Censo demográfico- Características da população e dos domicílios. Rio de Janeiro: IBGE; 2010.

22. Arruda PN, Lima A, Scalize PS. Gestão dos serviços públicos de água e esgoto e cobrança pelo seu uso nos municípios Goianos operados pelas prefeituras. Rev Ambient Água 2016; 11(2):362-376.

23. Scalize PS, Fernandes NC, Arruda PN, Ferreira NC, Cunha EH, Santos AF. Avaliação da turbidez em amostras de água tratada coletadas nos municípios do Estado de Goiás. Rev Monografias Ambient 2014; 13(3):3429-3436.

24. Yang YJ, Goodrich JA, Clark RM, Li SY. Modeling and testing of reactive contaminant transport in drinking water pipes: Chlorine response and implications for online contaminant detection. Water research 2008; 42(6):1397-1412.

25. Moimaz SAS, Saliba O, Chiba FY, Sumida DH, Garbin CAS, Saliba NA. Fluoride concentration in public water supply: 72 months of analysis. Braz Dent J 2012; 23(4):451-456.

26. Brasil. Portaria no 2.914 , de 12 de dezembro de 2011. Dispõe sobre os procedimentos e responsabilidades relativos ao controle e vigilância da qualidade da água para consumo humano e seu padrão de potabilidade. Diário Oficial da União 2011; 13 dez.

27. Companhia Ambiental do Estado de São Paulo (CETESB). Guia nacional de coleta e preservação de amostras: água, sedimento, comunidades aquáticas e efluentes líquidos. São Paulo: CETESB; 2011.

28. American Public Health Association (APHA), American Water Works Association (AWWA), Water Environment Federation (WEF). Standard methods for the examination of water and wastewater. $21^{\text {st }}$ ed. Washington: APHA, AWWA, WEF; 2005.

29. Estado de Goiás. Secretaria de Estado de Gestão e Planejamento. Sistema Estadual de Geoinformação. Dados climáticos de temperatura. [acessado 2015 Set 24] Disponível: http://www.sieg.go.gov.br/

30. Brasil. Portaria no 635, de 26 de dezembro de 1975. Aprova normas e padrões sobre fluoretação da água dos sistemas públicos de abastecimento, destinada ao consumo humano. Diário Oficial da União 1976; 30 jan.
31. Ramires I. Avaliação da concentração de flúor na água de abastecimento público, antes e depois dos procedimentos de fluoretação [dissertação]. Bauru: Faculdade de Odontologia de Bauru; 2004.

32. Panizzi M, Peres MA. Dez anos de heterocontrole da fluoretação de águas em Chapecó, Estado de Santa Catarina, Brasil. Cad Saude Publica 2008; 24(9):20212031.

33. Craig L, Lutz A, Berry KA, Yang W. Recommendations for fluoride limits in drinking water based on estimated daily fluoride intake in the Upper East Region, Ghana. Sci Total Environ 2015; 532:127-137.

34. Daré F, Dall'Aglio Sobrinho M, Libânio M. Avaliação do processo de fluoretação nos sistemas de abastecimento de água da região de Araçatuba, São Paulo. Eng Sanit Ambient 2009; 14(2):173-182.

35. Leivas LL, Tovo MF, Ardenghi TM, Feldens CA, Kramer PF, Faraco Junior IM. Heterocontrole da fluoretação das águas de abastecimento público do Município de Canoas/RS. Stomatos 2010; 16(30):11-20.

36. Buzalaf MA, Granjeiro JM, Damante CA, Ornelas F. Fluctuations in public water fluoride level in Bauru, Brazil. J Public Health Dent 2002; 62(3):173-176.

37. Galagan DJ, Vermillion JR. Determining optimum fluoride concentrations. Public Health Rep 1957; 72(6):491-493.

38. Ramadan A, Hilmi Y. The influence of climate on the determination of the upper permissible fluoride level in potable water in Sudan. Fluoride 2014; 47(2):170180.

39. Villa AE, Guerrero S, Villalobos J. Estimation of optimal concentration of fluoride in drinking water under conditions prevailing in Chile. Community Dent Oral Epidemiol 1998; 26(4):249-255.

Artigo apresentado em 17/03/2016

Aprovado em 12/11/2016

Versão final apresentada em 14/11/2016 